\title{
Reproducibilidad de espirometrías en trabajadores mexicanos y valores de referencia internacionales
}

José Rogelio Pérez-Padilla, M.C., ${ }^{(1)}$ Justino Regalado-Pineda, M. en C., ${ }^{(1)}$ Juan C arlos Vázquez-García, M. en C.(1)

\section{Pérez-Padilla JR, Regalado-Pineda J, Vázquez-García JC. Reproducibilidad de espirometrías en trabajadores mexicanos y valores de referencia internacionales. Salud Publica Mex 2001;43:113-121.} EI texto completo en inglés de este artículo está disponible en: http://www.insp.mx/salud/index.html

\section{Resumen}

Objetivo. Analizar la reproducibilidad de las espirometrías en un grupo de trabajadores mexicanos que demandaban incapacidad, así co mo determinar qué tan apropiados son los valores de referencia internacionales cuando se aplican en población mexicana. Material y métodos. Se estudiaron 5771 espirometrías realizadas en el Instituto $\mathrm{N}$ acional de Enfermedades Respiratorias de México con equipo y métodos recomendados por la Sociedad Americana del Tórax (ATS, por sus siglas en inglés). D el total de espirometrías, 685 se practicaron en mujeres. Se generaron ecuaciones de regresión múltiple para hombres y mujeres con base en edad y talla, y se les comparó con las notificadas para población estadunidense por Knudson y Hankinson, para europeos, por Q uanjer, y para mexicanos, por Rodríguez Reynaga. Resultados. El 80\% de las pruebas realizadas llenaban los criterios de reproducibilidad propuestos por la ATS, tanto para la capacidad vital forzada (FVC) como para el volumen espiratorio forzado en el primer segundo $\left(\mathrm{FEV}_{1}\right)$, mientras que $10 \%$ de ellas no mostraron reproducibilidad para ninguna de las dos mediciones. Los hombres estudiados mostraron en promedio una FVC 12\% por arriba del estándar de Q uanjer, 22\% por encima del de Knudson, $3 \%$ por arriba del de Hankinson y $6 \%$ superior al de Rodríguez Reynaga, mientras que las mujeres arrojaron

\author{
Pérez-Padilla JR, Regalado-Pineda J, \\ Vázquez-García JC. \\ Spirometric reproducibility \\ and reference values \\ in Mexican workers claiming disability. \\ Salud Publica Mex 2001;43:113-121. \\ The English version of this paper \\ is available at: http://www.insp.mx/salud/index.html
}

\begin{abstract}
A bstract
Objective. To decribe spirometric function and adjustment to foreign prediction equations in Mexican workers claiming work related disability. Material and methods. We reviewed 5771 spirometries done at the Mexican $\mathrm{N}$ ational Institute of Respirato ry D iseases performed with equipment and methods proposed by the American Thoracic Society. $W$ ith the spirometries we generated multiple regression equations separated for men and women based on age and height, compared to other in common use reported by Knudson and Hankinson in N orth A merica and by Q uanjer in europeans. Results. $80 \%$ of the tests were reproducible for FVC and FEV according to ATS, wheras $10 \%$ were reproducible for neither. Mean FVC in men was $12 \%$ above values reported by $Q$ uanjer, $22 \%$ above $K$ nudson, $3 \%$ above Hankinson and $6 \%$ above Rodriguez-Reynaga, whereas similar values for women were 18\%,10\%,0\% and 1\%. Excluding obese and those who had less than 2 acceptable maneuvers, the numbers increase by $1-2 \%$. FEV was also above predicted. Conclusions. Most workers requesting disability are able to generate a reproducible spirometry. However for the same gender, age and height, workers had a FEV and a FVC above normal values reported by Knudson and Q uanjer and are more similar to those reported by Hankinson in Mexican-A mericans. W hile a set of appropiate ref-
\end{abstract}

Trabajo presentado en la LXXXVIII Reunión de la Asociación de Investigación Pediátrica, junio de 1999, San José Iturbide, Guanajuato, México.

(1) Instituto $\mathrm{N}$ acional de Enfermedades Respiratorias, México.

Fecha de recibido: 25 de febrero de 2000 - Fecha de aprobado: 11 de diciembre de 2000

Solicitud de sobretiros: Dr. José Rogelio Pérez Padilla. Instituto N acional de Enfermedades Respiratorias,

Departamento de Fisiología Respiratoria. Calzada de Tlalpan 4502, 14080 México, D.F., México. Correo electrónico: perezpad@ servidor.unam.mx 
porcentajes de 18, 10, 0 y 1\%, respectivamente. Excluyendo a obesos y a los que no realizaron dos esfuerzos aceptables, los valores se incrementaban en 1 a $2 \%$. El FEV tuvo un comportamiento similar. Conclusiones. Las espirometrías de un alto porcentaje de trabajadores que demandan incapacidad, son reproducibles. Tienen, para la misma talla, edad y género, un FEV y una FVC mayores que los notificados por Knudson en Estados Unidos de América y por Q uanjer en Europa, aunque similares a los encontrados por Hankinson en méxicoamericanos. Mientras se obtienen valores de referencia confiables, las ecuaciones de regresión del grupo estudiado producen menos errores en la determinación de incapacidad en México. El texto completo en inglés de este artículo está disponible en: http://www.insp.mx/ salud/index.html

Palabras clave: espirometría; reproducibilidad de resultados; valores de referencia; México erence values are obtained, regression equations obtained from the studied group will generate less error in the evaluation of disability in mexican workers. The English version of this paper is available at: http://www.insp.mx/salud/ index.html

Key words: spirometry; reproducibility of results; reference values; Mexico
T a espirometría es la mejor prueba para evaluar la $\perp$ función mecánica pulmonar. Es la prueba más reproducible siempre y cuando se sigan lineamientos estrictos de calidad como los recomendados por la Sociedad Americana del Tórax (ATS, por sus siglas en inglés). ${ }^{1}$ Estos lineamientos incluyen criterios para la compra de espirómetros, para las maniobras a realizar y su aceptabilidad, así como para el análisis y la interpretación de las mismas. ${ }^{2}$ Por las razones mencionadas, la espirometría es una de las pruebas esenciales para valorar la incapacidad funcional laboral.

La reproducibilidad de la espirometría es crucial para interpretar los cambios en un mismo trabajador o en diferentes trabajadores. La variabilidad espirométrica se incrementa en niños y en ancianos en relación con los jóvenes, cuando se trata de sujetos sanos. También aumenta en enfermos, pero se cuenta con una información escasa, sobre la variabilidad en trabajadores no se conoce ninguna procedente de México, sobre todo hecha con estándares internacionales.

Un aspecto esencial de la interpretación espirométrica es la comparación con valores de referencia, paso indispensable cuando se carece de un seguimiento longitudinal de los trabajadores. Cuando los valores de referencia espirométricos son inadecuados, se generan errores en la interpretación. Los espirómetros son equipos importados que incluyen valores de referencia generados en poblaciones extranjeras y cuya aplicabilidad a la mexicana no está demostrada. Si los valores de referencia son demasiado elevados para los trabajadores mexicanos, se generarán falsos positivos, es decir, sujetos con función normal llamados anormales. Por otro lado, si los valores son demasiado bajos para la población, se generarán falsos negativos, es decir trabajadores con deficiente función pero que se diagnostican como normales. Una de las fuentes de variabilidad es el origen étnico de las personas, que contribuye hasta con $15 \%$ de la variabilidad espirométrica. ${ }^{2,3}$ Así, es posible que la población mexicana muestre algunas diferencias en la función espirométrica respecto a la de sujetos de origen europeo y estadunidense.

El objetivo del presente trabajo es analizar la reproducibilidad de las espirometrías realizadas en un grupo de trabajadores que demandaban incapacidad. Fueron estudiados con un estricto control de calidad y de acuerdo con lineamientos internacionales. Adicionalmente se determina qué tan apropiados son los valores de referencia utilizados frecuentemente para la población mexicana.

\section{Material y métodos}

Entre octubre de 1997 y julio de 1998, se estudió, en el laboratorio de Fisiología Respiratoria del Instituto Nacional de Enfermedades Respiratorias (INER), en la Ciudad de México, México, a 5771 sujetos enviados por la Junta de Conciliación y Arbitraje de la Secretaría del Trabajo, para que se les realizara una espirometría como parte de la evaluación de incapacidad laboral. No se tuvo conocimiento de los motivos de disputa en relación con la incapacidad laboral, ni de otros antecedentes de los trabajadores. El estudio de éstos se hizo en el marco de un convenio que incluía la realización de las pruebas solicitadas sin intervenir en la selección de las mismas. Las pruebas 
notificadas se hicieron en forma rutinaria (en promedio, se realizaron 80 diarias), por lo que no se tuvieron precauciones especiales relacionadas con un protocolo de investigación. También es conocido que la cooperación de los trabajadores en busca de incapacidad es irregular, ya que algunos buscan resultados bajos que apoyen sus demandas. Del grupo de sujetos estudiados, no se obtuvo información sobre la exposición al tabaco, el origen geográfico, ni sobre antecedentes más concretos de exposición laboral.

\section{Metodología para las espirometrías}

Se utilizó exclusivamente un espirómetro volumétrico con selle en seco (Sensormedics modelo 922, Sensormedics Co., Yorba Linda, California, Estados Unidos de América -EUA-) que cumple con los criterios de calidad recomendados por la ATS. ${ }^{1}$ Para calibrar el espirómetro, diariamente se usó una jeringa de tres litros (catálogo 762759, Sensormedics Co., Yorba Linda, California, EUA). Se siguieron las pautas de un manual de procedimientos para espirometrías, que establece detalles del uso del equipo y del control de calidad de las pruebas. Antes de cada estudio se registró el peso y la talla del trabajador, así como la temperatura ambiental. Los técnicos fueron capacitados en el INER, de acuerdo con los lineamientos del curso de espirometría de los Institutos Nacionales de Salud y Seguridad Ocupacional (NIOSH, por sus siglas en inglés), de Estados Unidos de América.

Se utilizaron los procedimientos estandarizados que sugiere la ATS. ${ }^{1}$ La aceptación o el rechazo de esfuerzos se hace automáticamente, y el software incluye mensajes de error basados en los criterios de calidad espirométrica, así como sugerencias al final de cada esfuerzo que facilitan la labor del técnico. Por otro lado, el técnico siempre supervisa las decisiones de la computadora con la posibilidad de corregirlas.

Por motivos de higiene, se registró sólo la porción espiratoria de la respiración forzada, para evitar que los sujetos inhalaran aire del espirómetro. Las boquillas eran desechables. Se pidió a los trabajadores que pasaran al laboratorio de función pulmonar en grupos de tres para que se familiarizaran con la prueba. Se les explicó el motivo del estudio y la técnica que se sigue para realizar la espirometría, misma que fue demostrada por el técnico. Se realizó un máximo de nueve maniobras espirométricas con el objetivo de completar tres aceptadas por el espirómetro, de acuerdo con los criterios de la ATS (lo que resultó en un código de error 000). Si el trabajador tendía a hacer una doble inspiración se solicitaba que repitiera el esfuerzo con la ayuda de pinzas nasales. Las espirometrías se hicieron manteniendo a los sujetos de pie.

Todos los esfuerzos (nueve maniobras espiratorias forzadas como máximo), se registraron en la memoria de la computadora. La base de datos espirométrica se genera automáticamente con un programa opcional de Sensormedics (Convert) que evita la captura manual. Se hizo una selección de las espirometrías sospechosas de error después de revisarlas con detenimiento. Se consideraron como indicadores de sospecha los valores extremos en las diferentes variables o la presencia de códigos de error.

La calidad de las espirometrías se valoró con base en varios criterios de uso frecuente. El número de maniobras aceptables por sujeto de acuerdo con criterios de la $\mathrm{ATS}^{1}$ puede ir de cero hasta tres; este último es el máximo que el programa espirométrico almacena y se le considera como el ideal.

Se utilizaron también las calificaciones de Enright $^{4,5}$ para espirometrías, basadas en la reproducibilidad intraprueba; de acuerdo con ello, la calificación A es la más alta y requiere de la presencia de tres esfuerzos aceptables, así como de una diferencia entre los dos mejores flujos espiratorios máximos (dPEFR) menor a $5 \%$, una diferencia entre los dos mejores volúmenes espiratorios forzados en el primer segundo $\left(\mathrm{dFEV}_{1}\right)$ menor a $5 \%$ o a $100 \mathrm{ml}$, una diferencia entre las dos mejores capacidades vitales forzadas (dFVC) menor a $3 \%$ y un tiempo espiratorio (FET100) mayor a seis segundos. Esto implica que existe una gran similitud entre las dos mejores maniobras de cada prueba. En el grado $B$ se requiere de tres esfuerzos aceptables, con una dPEFR menor a $10 \%$, una $\mathrm{dFEV}_{1}$ menor a $5 \%$ o $100 \mathrm{ml}$, una dFVC menor a 5\% y un FET100 mayor o igual a seis segundos. Para el grado $C$ se necesitan dos esfuerzos aceptables, y una dPEFR menor a $10 \%$, un FET100 mayor o igual a seis segundos, una dFVC menor a $5 \%$ y cualquier $\mathrm{dFEV}_{1}$. Para la calificación D se requiere de dos esfuerzos aceptables, con cualquier dPEFR, $\mathrm{dFEV}_{1}$, FET100 mayor o igual a seis segundos o dFVC. El grado E se asigna cuando sólo un esfuerzo es aceptable, y el F, cuando ninguno lo es. Los criterios de Enright y colaboradores son más estrictos que los de la ATS, ya que se añade a la reproducibilidad del volumen espiratorio forzado en el primer segundo $\left(\mathrm{FEV}_{1}\right)$ y de la capacidad vital forzada (FVC), la del flujo espiratorio máximo (PEFR), mucho más difícil de lograr; además se separa la calificación para la reproducibilidad de flujos y volúmenes. Para la calificación de los flujos se utilizó la clasificación original, pero para volúmenes se le modificó ligeramente; así, se consideró la categoría A, cuando el promedio del tiempo 
para llegar a la capacidad vital (FET100) -medida disponible en el espirómetro utilizado- alcanzaba los seis segundos o más en todos los esfuerzos hechos. Con base en la clasificación original se determinó un tiempo espiratorio mayor a 10 segundos para todas las pruebas aceptadas. El FET100, frecuentemente es menor al tiempo espiratorio, ya que se logra llegar a la capacidad vital antes de parar la espiración. Es importante hacer notar que cumplir con los criterios de Enright y colaboradores es más difícil con el espirómetro utilizado, debido a que no incluye los mensajes apropiados para cumplirlos. La categoría $C$ de Enright equivale a la 2 de la ATS.

Se valoró la reproducibilidad de los esfuerzos para $\mathrm{FEV}_{1}$ y FVC. Se consideraron reproducibles cuando los dos mejores esfuerzos no difirieron en más de $200 \mathrm{ml}$ en ambas. Se consideró una reproducibilidad de 0 , si tanto el $\mathrm{FEV}_{1}$ como la FVC reunían criterios de reproducibilidad; de 1, si sólo una de los dos pruebas cumplía con dichos criterios, y de 2, si ninguna lo hacía.

Como medidas de reproducibilidad se utilizaron además, la diferencia entre las dos mejores mediciones de $\mathrm{FEV}_{1}, \mathrm{FVC}, \mathrm{FEV}_{1} / \mathrm{FVC}$, del flujo máximo instantáneo al $50 \%$ de la capacidad vital $\left(\operatorname{Vmax}_{50}\right)$ o al $25 \%$ de la capacidad vital $\left(\operatorname{Vmax}_{25}\right)$, o bien el flujo mesoespiratorio $\left(\mathrm{FEF}_{25-75}\right)$, tanto en números absolutos (litros o litros/segundo) como en valor porcentual (diferencia entre los dos mejores por 100/valor más alto). Este último valor porcentual ajusta por el tamaño pulmonar.

\section{Análisis de los datos}

Se hicieron estadísticas descriptivas de los datos con medias y desviaciones estándar. Para fines de interpretación, se consideraron, como variables más importantes y medidas más reproducibles, el $\mathrm{FEV}_{1}$ y la FVC junto con su cociente.

Para las ecuaciones de predicción se elaboraron los modelos de regresión múltiple más importantes, separadamente para hombres y mujeres, y con base en edad y talla; estos modelos en general se incluyen en las ecuaciones de referencia. Se generaron ecuaciones diferentes para hombres y mujeres con el fin de evitar presumir que el efecto del género es un desplazamiento paralelo y que las pendientes de función pulmonar en relación con la talla y el peso son similares en población masculina y femenina. ${ }^{6}$ Se compararon los valores espirométricos del presente estudio con los notificados para poblaciones estadunidenses por Knudson y colaboradores $^{7}$ y para europeos por Quanjer y colaboradores, ${ }^{8}$ así como los más recientes que incluyen ecuaciones separadas para estadunidenses caucásicos, negros y de origen mexicano. ${ }^{9}$
En México se cuenta con un estudio previo de valores de referencia espirométrica hecho por Rodríguez Reynaga y colaboradores, y que es utilizado ampliamente en la medicina laboral (estudio no publicado). Con las tablas disponibles, para el presente trabajo se volvieron a generar las ecuaciones de regresión lineal incluyendo talla y edad, aunque no se midió el $\mathrm{FEV}_{1}$. Se incluyeron modelos con el índice de masa corporal y con indicadores de obesidad o de una calidad espirométrica pobre para investigar su impacto en la función respiratoria.

\section{Resultados}

Se concentraron espirometrías de 5086 hombres y de 685 mujeres. La edad promedio para el grupo estudiado fue de 51 años. Las pruebas se aplicaron a una temperatura ambiente de entre 19 y $25^{\circ} \mathrm{C}$, con una presión barométrica promedio de $583 \mathrm{mmHg}$ y a una altura de $2240 \mathrm{msnm}$.

El cuadro I muestra los datos antropométricos de la población estudiada de acuerdo con el género. Se puede observar que tanto el $\mathrm{FEV}_{1}$ como la FVC y la mayoría del resto de mediciones espirométricas son un poco mayores en hombres que en mujeres, lo que en gran parte se debe al mayor tamaño corporal de aquéllos. Del total de trabajadores, 1595 (27.6\%) tenían un índice de masa corporal mayor a 30 que define obesidad, y 1197 (20.74\%) tenían menos de dos esfuerzos espirométricos aceptables.

En el cuadro I se muestran además algunos indicadores de la calidad de las espirometrías; en ese mismo cuadro se pueden observar los datos de variabilidad espirométrica al igual que en el cuadro II. En general la variabilidad es menor y la calidad mayor en los hombres que en las mujeres, pero en ambos grupos la mayoría de las pruebas es de alta calidad. La reproducibilidad de las pruebas de acuerdo con los criterios de la ATS no difirió entre hombres y mujeres. Por otro lado, los hombres tuvieron más frecuentemente tres esfuerzos aceptables, más pruebas con calificación A de Enright y menos pruebas con calificación $\mathrm{C}$ que las mujeres. Las pruebas aceptables no difirieron, por décadas de edad, ni en el número de pruebas reproducibles en el grupo completo ni en hombres o mujeres por separado. En cambio, el número de pruebas con calificación A de Enright es mayor en edades más tempranas que en edades más avanzadas, tanto en el grupo completo como en hombres.

La variabilidad intraprueba del $\mathrm{FEV}_{1}$ (diferencia en $\mathrm{ml}$ entre los dos mejores esfuerzos, $\mathrm{dFEV}_{1}$ ), no se vio afectada ni por el género ni por la talla, pero tendió a disminuir con la edad y a aumentar con el peso. Si se 
Cuadro I

Promedios y desviaciones estándar de LAS VARIABLES ESTUDIADAS EN POBLACIÓN TRABAJADORA DE ACUERDO CON EL GÉNERO. MÉXICO, 1997-1998
Variable

\begin{tabular}{|c|c|c|}
\hline Edad & $49.3 \quad(8)$ & $(8.5)$ \\
\hline Talla (cm) & $(5.7)$ & $(6)^{*}$ \\
\hline Peso (kg) & $65 \quad(11)$ & $74 \quad(12)^{*}$ \\
\hline Indice de masa corporal $\left(\mathrm{kg} / \mathrm{m}^{2}\right)$ & $28.4 \quad(4.6)$ & $28.0 \quad(4)$ \\
\hline FET $100(s)$ & $7.8 \quad(1.9)$ & $8.1(2.0)^{*}$ \\
\hline$\overline{\mathrm{FEV}} \mathrm{I}_{1}(\mathrm{I})$ & $2.4 \quad(0.5)$ & $3.3(0.7)^{*}$ \\
\hline FVC (I) & $2.97(0.6)$ & $4.2(0.7)^{*}$ \\
\hline PEFR (I/S) & $6.4 \quad(1.38)$ & $9.1(1.9)^{*}$ \\
\hline$\overline{\mathrm{FEV}_{1} / \mathrm{FVC}(\%)}$ & $80.6 \quad(6.2)$ & $79.7 \quad(6.5)$ \\
\hline $\operatorname{Vmax}_{50}(1 / s)$ & $3.9 \quad(1.2)$ & $4.9 \quad(1.7)^{*}$ \\
\hline $\operatorname{Vmax}_{25}(I / s)$ & $1.08(0.5)$ & $1.4(0.7)^{*}$ \\
\hline $\mathrm{FEF}_{2575}(\mathrm{l} / \mathrm{s})$ & $2.9 \quad(1.0)$ & $3.7 \quad(1.4)^{*}$ \\
\hline $\mathrm{dFVC}^{\ddagger}$ & $3.9 \quad(5.7)$ & $2.9(3.5)^{*}$ \\
\hline $\mathrm{dFEV}_{1}^{\ddagger}$ & $3.7 \quad(4.8)$ & $3.2(3.9)^{*}$ \\
\hline $\mathrm{dPEFR}^{\ddagger}$ & $7.5 \quad(8.8)$ & $6.6 \quad(9.2)^{*}$ \\
\hline $\mathrm{dVmax}_{50}{ }^{\ddagger}$ & $9.1 \quad(9.2)$ & $9.1 \quad(8.6)$ \\
\hline $\mathrm{dV}_{\max }{ }_{25}^{\ddagger}$ & $14.1(12.3)$ & $13.4(11.9)$ \\
\hline $\mathrm{dFEF}_{25-75^{\ddagger}}$ & $9.2 \quad(9.3)$ & $8.9 \quad(8.4)$ \\
\hline
\end{tabular}

* Diferencias significativas $(p<0.05)$, en general, debidas a diferencias en tamaño entre hombres y mujeres

₹ Estimaciones de la variabilidad intraprueba intrasujeto. Representan la diferencia porcentual entre las dos mejores mediciones en el mismo sujeto y ocasión

FET 100: tiempo para llegar a la capacidad vital

$\mathrm{FEV}_{1}$ : volumen espiratorio forzado en el primer segundo

FVC: capacidad vital forzada

PEFR: flujo espiratorio máximo

$V_{\max }$ : flujo instantáneo al $50 \%$ de la capacidad vital

$V \max _{25}$ : flujo instantáneo al $25 \%$ de la capacidad vital

$\mathrm{FEF}_{25-75}$ : flujo promedio entre 25 y $75 \%$ de la capacidad vital

$\operatorname{Vmax}_{50}$ y V $\max _{25}$ : flujo instantáneo al 50 y al $25 \%$ de la capacidad vital

expresa como valor porcentual (diferencia en $\mathrm{ml}$ dividido entre el valor máximo), se va incrementando con la edad y el peso y disminuyendo con la talla. Para la FVC, la variabilidad en $\mathrm{ml}$ aumenta con la talla y el peso y tiende a bajar en los hombres, sin que exista relación con la edad. Si se expresa porcentualmente, se incrementa con la edad y el peso, y baja en los hombres, aunque no se afecta con la talla. La variabilidad es, por lo tanto, una función compleja de la edad y de las características antropométricas, y no se derivan ventajas claras de expresarla en $\mathrm{ml}$ o en forma porcentual.

En el cuadro III se describen las ecuaciones de regresión obtenidas con nuestros resultados, así como

\section{Cuadro II \\ Calidad espirométrica en trabajadores \\ MEXICANOS QUE DEMANDABAN INCAPACIDAD De ACUerdo Con el GÉnero. MÉxico, 1997-1998}

Criterio de calidad

$$
\frac{\text { Mujeres }}{n \quad(\%)} \quad \frac{\text { Hombres }}{n \quad(\%)}
$$

Reproducibilidad de flujos, calificación de Enright*

\begin{tabular}{rrrrrr}
$A$ & 135 & $(19.7)$ & 1293 & $(25)$ & \\
\hline$B$ & 54 & $(8)$ & 547 & $(11)$ & \\
\hline$C$ & 120 & $(18)$ & 935 & $(18)$ & \\
\hline$D$ & 203 & $(30)$ & 1287 & $(25)$ & \\
\hline$E$ & 113 & $(17)$ & 727 & $(14)$ & \\
\hline$F$ & 60 & $(9)$ & 297 & $(6)$ & $p<0.0001$
\end{tabular}

Reproducibilidad de volúmenes, calificación de Enright*

\begin{tabular}{rrrrrr}
$A$ & 173 & $(25)$ & 1671 & $(33)$ & \\
\hline$B$ & 38 & $(6)$ & 380 & $(7)$ & \\
\hline$C$ & 143 & $(21)$ & 1061 & $(21)$ & \\
\hline$D$ & 158 & $(23)$ & 947 & $(19)$ & \\
\hline$E$ & 158 & $(23)$ & 727 & $(14)$ & \\
\hline$F$ & 60 & $(9)$ & 297 & $(6)$ & $p<0.0001$
\end{tabular}

Reproducibilidad espirométrica, criterios de la ATS

\begin{tabular}{lrlrll} 
En FVC y FEV & 541 & $(80)$ & 3948 & $(78)$ & \\
\hline En uno de los dos & 73 & $(11)$ & 592 & $(12)$ & \\
\hline En ninguno & 71 & $(10)$ & 546 & $(11)$ & $p=0.7$
\end{tabular}

$\mathrm{N}$ úmero de pruebas aceptables de acuerdo con criterios de la ATS*

\begin{tabular}{rrrrrr}
3 & 307 & $(45)$ & 2641 & $(52)$ & \\
\hline 2 & 205 & $(30)$ & 1421 & $(28)$ & \\
\hline 1 & 113 & $(17)$ & 727 & $(14)$ & \\
\hline 0 & 60 & $(9)$ & 297 & $(6)$ & $0<0.001$
\end{tabular}

* Distribuciones estadísticamente diferentes entre hombres y mujeres por prueba de $\chi^{2}$

ATS: Sociedad Torácica A mericana

FVC: capacidad vital forzada

$\mathrm{FEV}_{1}$ : volumen espiratorio forzado en el primer segundo

su coeficiente de determinación $\left(R^{2}\right)$ y el error estándar de la estimación. Es notable que la capacidad para predecir el $\mathrm{FEV}_{1}$ y la FVC es mejor que para las otras mediciones. Especialmente mala es la predicción del $V_{\max _{50}}$ por lo que no es recomendable su uso. Mientras que para $\mathrm{FEV}_{1}$ y FVC la conversión a porcentaje del predicho reduce considerablemente el coeficiente de variación (de 17-18\% a 12-13\%), esto no sucede con el Vmax ${ }_{50}$ y poco con el PEFR.

Las variables espirométricas tienen una relación lineal directa con la talla e inversa con la edad. La relación $\mathrm{FEV}_{1} / \mathrm{FVC}$ decae con la edad, pero no cambia sustancialmente con la talla. En las mujeres no se detectó un impacto adverso de la obesidad en el $\mathrm{FEV}_{1} \mathrm{o}$ la FVC, pero los sujetos con mala calidad espirométrica tenían una FVC menor. En hombres, un índice de masa corporal mayor de 30 (obesidad) se asociaba a una baja de $112 \mathrm{ml}$ del $\mathrm{FEV}_{1}$, y uno mayor de 40 se acompañaba de una caída de $600 \mathrm{ml}$, mientras que 


\section{Cuadro III \\ ECUACIONES DE PREDICCIÓN ESPIROMÉTRICA EN TRABAJADORES MEXICANOS QUE DEMANDABAN INCAPACIDAD. MÉXICO, 1997-1998}

$\begin{array}{llllll}\text { Variable dependiente } & \begin{array}{c}\text { Coeficiente } \\ \text { talla }(\mathrm{cm})\end{array} & \begin{array}{c}\text { Coeficiente } \\ \text { edad(años) }\end{array} & \begin{array}{l}\text { Cons- } \\ \text { tante }\end{array} & R^{2} \quad \text { EEE }\end{array}$

Mujeres

\begin{tabular}{|c|c|c|c|c|c|}
\hline $\mathrm{FEV}_{1}(\mathrm{I})$ & 0.0288 & -0.0269 & -0.6354 & 0.43 & 0.33 \\
\hline FVC (I) & 0.0374 & -0.0274 & -1.3607 & 0.42 & 0.38 \\
\hline $\operatorname{Vmax}_{50}(\mathrm{l} / \mathrm{s})$ & 0.0217 & -0.0463 & 2.9431 & 0.12 & 1.09 \\
\hline $\operatorname{Vmax}_{25}(\mathrm{I} / \mathrm{s})$ & 0.010 & -0.0242 & 0.7684 & 0.16 & 0.47 \\
\hline $\mathrm{FEF}_{25-75}(\mathrm{l} / \mathrm{s})$ & 0.0216 & -0.0472 & 1.9704 & 0.19 & 0.84 \\
\hline $\mathrm{FEV}_{1} / \mathrm{FVC}(\%)$ & NO & -0.1594 & 89.09 & 0.08 & 4.5 \\
\hline
\end{tabular}

Hombres

\begin{tabular}{|c|c|c|c|c|c|}
\hline $\mathrm{FEV}_{1}(\mathrm{I})$ & 0.0398 & -0.0314 & -1.5642 & 0.41 & 0.47 \\
\hline FVC (I) & 0.0539 & -0.0289 & -3.1713 & 0.39 & 0.55 \\
\hline $\operatorname{Vmax}_{50}(\mathrm{I} / \mathrm{s})$ & 0.0317 & -0.0568 & 2.644 & 0.12 & 1.5 \\
\hline $\operatorname{Vmax}_{25}(\mathrm{I} / \mathrm{s})$ & 0.0126 & -0.0297 & 0.8669 & 0.17 & 0.61 \\
\hline $\mathrm{FEF}_{25-75}(\mathrm{l} / \mathrm{s})$ & 0.0274 & -0.0576 & 2.184 & 0.18 & 1.16 \\
\hline $\mathrm{FEV}_{1} / \mathrm{FVC}(\%)$ & no & -0.1926 & 89.95 & 0.09 & 5.1 \\
\hline $\mathrm{FEV}_{1} / \mathrm{FVC}(\%)$ & -0.0768 & -0.2033 & 103.0 & 0.1 & 5.09 \\
\hline
\end{tabular}

EEE: error estándar de la estimación

$\mathrm{FEV}_{1}$ : volumen espiratorio forzado en el primer segundo

FVC: capacidad vital forzada

$\operatorname{Vmax}_{50}$ : flujo instantáneo al $50 \%$ de la capacidad vital

$\operatorname{Vmax}_{25}$ : flujo instantáneo al $25 \%$ de la capacidad vital

$\mathrm{FEV}_{25-75}$ : flujo promedio entre 25 y $75 \%$ de la capacidad vital

Nota: estos cálculos se hicieron sin los sujetos con $\mathrm{FEV}_{1} / \mathrm{FVC}$ de $100 \%$ y los que tienen $\mathrm{FEV}_{1} / \mathrm{FVC}$ menos de $1.64 \mathrm{DE}$ abajo del predicho. Son muy parecidos a los calculados con todos los trabajadores

para la FVC las caídas equivalentes fueron de $209 \mathrm{ml}$ y $814 \mathrm{ml}$, respectivamente. Los hombres que no pudieron hacer un mínimo de dos esfuerzos aceptables mostraron una reducción tanto del $\mathrm{FEV}_{1}$ como de la FVC.

En la figura 1 puede verse que los valores normales europeos de Quanjer no ajustan bien a la población de trabajadores mexicanos estudiados, lo mismo que los estadunidenses de Knudson y Hankinson. En caso de un ajuste exacto a los valores de referencia extranjeros en todas las tallas, se esperarían líneas horizontales en la marca de $100 \%$. El grado de desviación de $100 \%$ es una medida de la discrepancia con los valores de referencia extranjeros. Las gráficas del lado izquierdo tienen los datos de las mujeres, y las de la derecha, los de los hombres. En las gráficas superiores se describe el $\mathrm{FEV}_{1}$ como porcentaje del predicho por Quanjer (línea inferior) y por Knudson (línea superior). Puede observarse que ambas líneas, representación de las medianas, están por encima de la horizontal del 100\%. Adicionalmente, en hombres se demuestra una predicción mucho más errónea a tallas bajas para la ecuación de Knudson. Los predichos por Hankinson en mexicoamericanos (gráficas inferiores) están bastante cercanos al $100 \%$, sobre todo en mujeres para $\mathrm{FEV}_{1}$ y FVC, y el mayor error en hombres de talla baja no desaparece. Las oscilaciones en los extremos son por la escasez de datos en la zona. El comportamiento de la FVC es similar al descrito en la figura para el $\mathrm{FEV}_{1}$ (cuadro IV). Los valores mexicanos de Rodríguez Reynaga ajustan mejor que los extranjeros aun cuando no notifican $\mathrm{FEV}_{1}$, como se muestra numéricamente en el cuadro IV. Los valores señalados por Hankinson en méxicoamericanos ${ }^{9}$ muestran un ajuste aceptable, pero aun cuando se excluya a los sujetos con menos de dos esfuerzos válidos y a los obesos -criterio frecuente en los estudios para generar valores de referencia-, dichos valores todavía quedan por arriba de $100 \%$, especialmente en hombres (cuadro IV).

El tipo de valor de referencia que se escoja determina el número de sujetos considerados fuera del rango normal o anormales. Frecuentemente se utiliza como límite inferior de la normalidad el $80 \%$ del valor predicho, aunque el límite estadísticamente más confiable es el de 1.64 desviaciones estándar (DE) por debajo del promedio. En los trabajadores estudiados, 309 resultaron con un $\mathrm{FEV}_{1}$ menor a $1.64 \mathrm{DE}$ del promedio notificado por Quanjer; 124 sujetos con $\mathrm{FEV}_{1}$ menor al predicho por Knudson; y 433, por debajo de los predichos en el presente estudio. Un mayor número resulta anormal si se considera como límite el cuestionable $80 \%$ del predicho. Para la FVC, 201 trabajadores quedaron por debajo del límite inferior predicho por Rodríguez Reynaga y 382 por debajo del predicho en este estudio. En total 843 trabajadores estaban por debajo del límite inferior predicho por el presente estudio, considerando ya sea el $\mathrm{FEV}_{1}$, la FVC o el cociente entre ambos. Utilizando las ecuaciones provenientes del grupo de trabajadores estudiado, se incrementa considerablemente el número de sujetos con una función anormalmente baja y, por lo tanto, el número de trabajadores con criterios funcionales de incapacitación.

\section{Discusión}

Sin duda el presente estudio tiene limitaciones que conviene tener en cuenta para interpretar adecuadamente los resultados. No se obtuvo información sobre el tabaquismo y la exposición laboral, ni tampoco sobre el tipo de síntomas respiratorios, los datos arrojados por la exploración física, los hallazgos radiológicos 

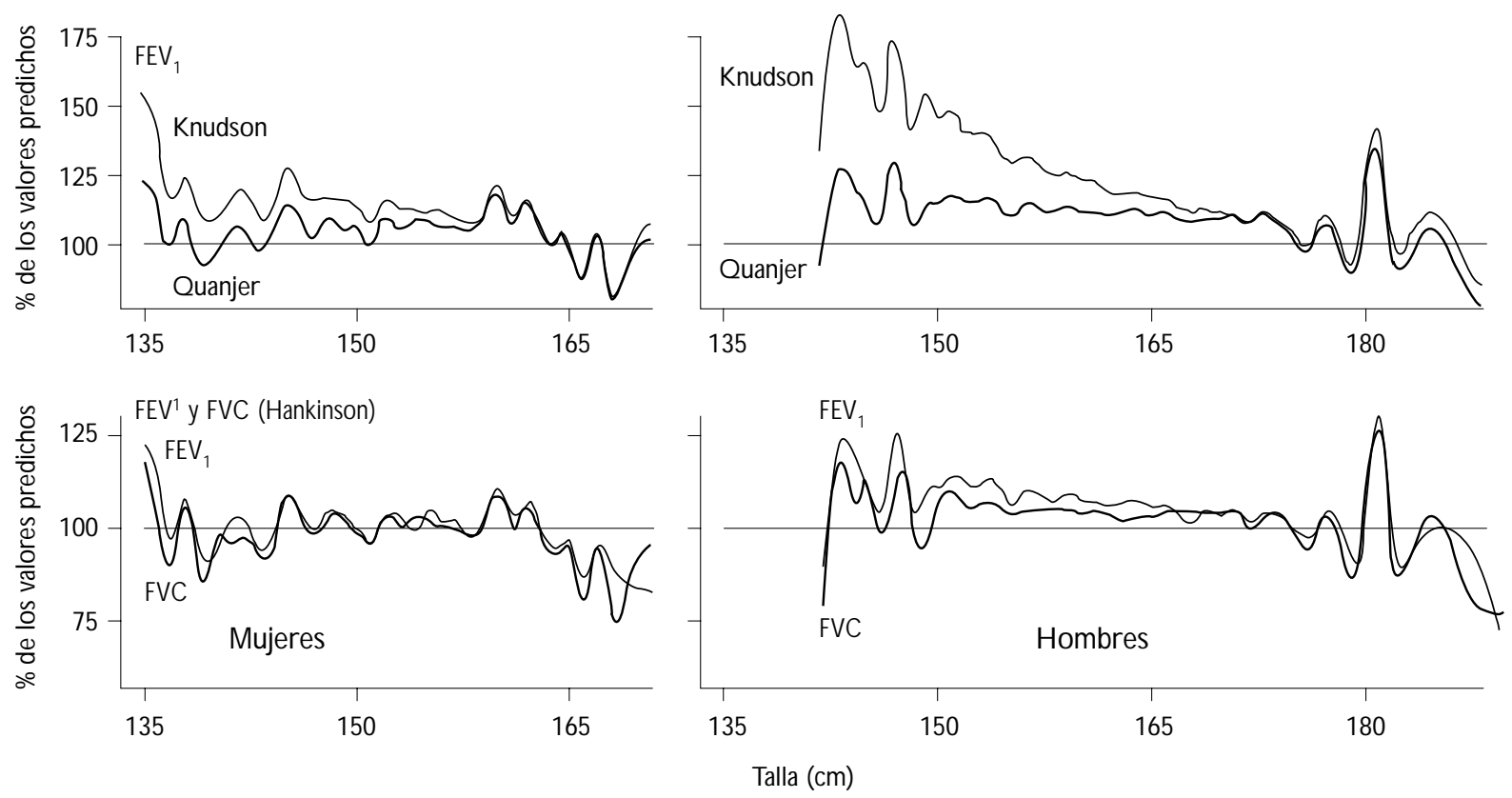

Figura 1. Volumen espiratorio forzado en el primer segundo como porcentaje de los valores predichos EUROPEOS Y ESTADUNIDENSES, en trabajAdores MeXICANOS QUE DEMANDABAN INCAPACIDAD. MéXICO, 1997-1998

y la queja concreta. Varios de los aspectos previos pueden afectar adversamente la función respiratoria y su variabilidad. El impacto del sitio de residencia y de una posible exposición a la contaminación atmosférica en la función pulmonar es más débil, comparado con el del tabaquismo y las exposiciones laborales más perjudiciales. Por lo mismo, en la población estudiada existe un número desconocido de trabajadores afectados en la función respiratoria y la reproducibilidad espirométrica. Sin embargo, éstos constituyen un numeroso grupo que describe la función espirométrica en trabajadores que demandan incapacidad, cada vez mayor y del cual se desconocen muchas características. Adicionalmente, la información obtenida, a pesar de sus limitaciones, es útil para determinar el ajuste de los valores espirométricas de nuestra población a valores de referencia extranjeros de uso común usando una metodología aceptada internacionalmente. La calidad de las espirometrías realizadas es comparable con la encontrada en otros estudios epidemiológicos en trabajadores. Es importante precisar que la estandarización de la espirometría se inicia con la selección del equipo y sigue con la unificación del procedimiento, del análisis, de la selección de valores de referencia y de la interpretación. ${ }^{1,2}$ La variabilidad intrasujeto de la función respiratoria fue aceptable, a pesar de que provenía de trabajadores que buscaban compensación y de que las pruebas se realizaron en condiciones de trabajo intensivo y rutinario. La raza es una de las fuentes conocidas de variación espirométrica. Por ejemplo, con la misma talla, edad y sexo, los negros estadunidenses tienen valores de $\mathrm{FEV}_{1}$ y una FVC $12 \%$ menor que los de los blancos. Se considera que buena parte de esta diferencia (aunque no toda) se puede explicar por la relación entre la longitud torácica y la talla.

Llama la atención que los valores espirométricos encontrados están por arriba de los esperados para la población europea ${ }^{8}$ y la americana, ${ }^{7}$ con la misma talla y edad, a pesar de incluir un número desconocido de personas expuestas al tabaquismo o a riesgos respiratorios, a sujetos con un desempeño espirométrico poco confiable y a obesos. Dichos valores están incluso un poco por arriba de los valores mexicanos de Rodríguez Reynaga y colaboradores (no publicados) y de los notificados por Hankinson en mexicoamericanos, ${ }^{9}$ aunque éstos ajustan mucho mejor. Esto hace indispensable utilizar equipos y procedimientos vigentes para generar valores de referencia apropiados para la población mexicana. Hallazgos similares a los del presente estudio se han descrito en la población chilena ${ }^{10} \mathrm{y}$ aunque no se ha evaluado formalmente, ello pudiera 
derivarse de que en la talla de los chilenos el tronco guarda una mayor proporción, en comparación con las de otras poblaciones. La inclusión de enfermos, fumadores, obesos y personas que ejecutan pobremente la espirometría, así como de sujetos con un intervalo limitado de edades, puede explicar los bajos coeficientes de determinación alcanzados por las ecuaciones de predicción.

$\mathrm{Al}$ usar los valores de referencia incluidos en los espirómetros, es decir, valores espirométricos de ori- gen extranjero, se puede esperar un mayor número de falsos negativos en la identificación de trabajadores incapacitados, lo que va en su perjuicio. El estudio ideal para obtener ecuaciones de referencia es aquel que parte de un muestreo de población general, lo que es muy laborioso y costoso, y no aquel que se basa en una encuesta de trabajadores activos, habitualmente más sanos y resistentes que los que dejan de trabajar. La utilización de los valores de referencia para trabajadores, descritos en el cuadro IV, generarían menos

\section{Valores espirométricos Según género, como porcentaje de VARIOS VAlores de Referencia. Trabajadores MEXICANOS QUE DEMANDABAN INCAPACIDAD. México 1997-1998}

\begin{tabular}{|c|c|c|c|}
\hline Origen (referencia) & Variable & $\begin{array}{l}\text { Mujeres } \\
(n=685)\end{array}$ & $\begin{array}{l}\text { Hombres } \\
(n=5086)\end{array}$ \\
\hline & & $\begin{array}{ll}\bar{X} & (D E)\end{array}$ & $\begin{array}{ll}\bar{X} & (D E)\end{array}$ \\
\hline Europeo (Q uanjer) & $\mathrm{FEV}_{1} \%$ & 112 (19) & $109 \quad(18)$ \\
\hline & FVC\% & $118 \quad(18)$ & $112 \quad(17)$ \\
\hline & $\mathrm{FEV}_{1} / \mathrm{FVC}_{0} \%$ & $101 \quad(7.6)$ & $102 \quad(8)$ \\
\hline & $V \max 50 \%$ & $106 \quad(32)$ & $114 \quad(37)$ \\
\hline & Vmax $25 \%$ & $73 \quad(34)$ & $88 \quad(41)$ \\
\hline & $\mathrm{FEF}_{25-75} \%$ & $91 \quad(29)$ & $100 \quad(34)$ \\
\hline Estadunidense (Knudson) & $\mathrm{FEV}_{1} \%$ & $105 \quad(17)$ & $118 \quad(21)$ \\
\hline & FVC\% & $110 \quad(16)$ & $122 \quad(21)$ \\
\hline & $\mathrm{FEV}_{1} / \mathrm{FVC} \%$ & $95.6 \quad(7)$ & $97.9(8)$ \\
\hline Mexicano (Rodríguez Reynaga) ${ }^{*}$ & FVC (\%) & $101 \quad(16)$ & $106 \quad(16)$ \\
\hline & $\mathrm{FEF}_{25-75} \%$ & $76.2(25)$ & $81 \quad(28)$ \\
\hline Estadunidense (Hankinson) ${ }^{\ddagger}$ & FEV1 (\%) blancos & $99.4(16)$ & $104.3(17)$ \\
\hline & FVC (\%) blancos & $98.6(15)$ & $101.8(15)$ \\
\hline & FEV1/FVC blancos & $100.3(8)$ & $102.8 \quad(8.1)$ \\
\hline & FEV1 (\%) mexicanos & $100.7(16)$ & $105.1(17)$ \\
\hline & FVC (\%) mexicanos & $100.7(15)$ & $102.9(15)$ \\
\hline & FEV1/FVC (\%) mexicanos & $99.2(7.5)$ & $101.0 \quad(8)$ \\
\hline Estadunidense $(\text { Hankinson })^{\S}$ & FEV1 (\%) blancos & $101 \quad(15)$ & $105.3(17)$ \\
\hline & FVC (\%) blancos & $101 \quad(14)$ & $103.9(15)$ \\
\hline & FEV1/FVC blancos & $99.6 \quad(7)$ & $101.6 \quad(8.4)$ \\
\hline & FEV1 (\%) mexicanos & $102.6(15)$ & $106.0(17)$ \\
\hline & FVC (\%) mexicanos & $103.3(14)$ & $104.9(15)$ \\
\hline & FEV1/FVC (\%) mexicanos & $98.42(7.2)$ & $99.8 \quad(8.2)$ \\
\hline
\end{tabular}

\footnotetext{
* Rodríguez Reynaga y colaboradores no estudiaron el FEV1. A simismo, no reportan el error estándar de la estimación por lo que se utilizaron los descritos por Q uanjer

₹ Incluye a todos los trabajadores

$\S$ Datos excluyendo a los trabajadores con menos de dos esfuerzos espirométricos aceptables y a los obesos con índice de masa corporal mayor a 30 DE: desviación estándar

Nota: si la población de trabajadores fuera idéntica a la de referencia el valor esperado sería de $100 \%$. La terminación \% se refiere a los valores como porciento del predicho

Fuente: referencias 7-9
} 
errores que los de uso común, a pesar de que el impacto del tabaquismo y de las exposiciones laborales no está identificado y debido a que arrojan valores más altos que los estadunidenses o europeos. Una mejor opción que la utilización de valores de referencia internacionales, consiste en usar temporalmente los valores notificados por Hankinson, ${ }^{9}$ tomados de una muestra de la población general de mexicoamericanos. La media de los niños escolares de la ciudad de México se encuentra también sobre el promedio de los niños estadunidenses, incluyendo el de los mexicoamericanos. ${ }^{11}$

El objetivo principal de los programas de control de calidad es disminuir el error de medición; en el presente estudio éste se manifiesta como variabilidad intraobservador intraprueba. La calidad de las pruebas tiende a mejorar con el tiempo. Por ejemplo, hasta $15 \%$ de trabajadores no llenan los criterios de reproducibilidad de la ATS al primer intento; ${ }^{5}$ en el presente estudio la proporción fue de $20 \%$. Por otro lado, de 6000 trabajadores de 30 plantas estadunidenses, $93 \%$ tuvo en la tercera evaluación una calificación de flujo o volumen A o B, y 66\%, una calificación de flujo A, de acuerdo con los criterios de Enright. ${ }^{5}$ Estos porcentajes son mucho mayores que los encontrados entre los trabajadores mexicanos durante la primera prueba (35 y $22 \%$, respectivamente). Sin embargo, tomando los criterios de reproducibilidad más recientemente establecidos por la ATS, $80 \%$ de los trabajadores presentan una FVC y un $\mathrm{FEV}_{1}$ reproducibles, y aproximadamente $10 \%$ tiene una calidad espirométrica mala. Es de notar que estos resultados no descartan la utilidad de esas pruebas, ya que se ha visto que los sujetos con peor reproducibilidad tienen en el futuro una mayor morbilidad y mortalidad. Es decir, se trata de sujetos con mayor susceptibilidad a la enfermedad, quizá por hiperreactividad bronquial, pérdida de motivación, enfermedad pulmonar o debilidad muscular. También es de llamar la atención la diferencia entre la reproducibilidad que se puede lograr en condiciones rutinarias y con una carga de trabajo alta y la que se puede obtener en condiciones óptimas y con más tiempo, o dentro de un proyecto de investigación.

En conclusión, se puede afirmar que un grupo de trabajadores mexicanos evaluados espirométricamente, como parte de un estudio de incapacidad, mostraron valores por encima de los esperados para la talla, el género y la edad de sujetos europeos y estadunidenses. Los valores referidos se observan a pesar de que en el grupo estudiado se incluyó a sujetos con una buena o mala calidad espirométrica, a obesos, así como un número desconocido de fumadores y de expuestos a ambientes adversos para la respiración. Esto puede representar una diferencia étnica, en cuanto a la presencia de un tórax mayor, en los trabajadores mexicanos, para la misma talla o longitud de las piernas. Por lo tanto, la valoración de incapacidad funcional, con base en la comparación con ecuaciones de referencia europeas o estadunidenses, perjudica a los trabajadores mexicanos, ya que de esta manera, para ser clasificados como incapacitados, requieren de más pérdidas funcionales que si se utilizaran ecuaciones apropiadas.

\section{Referencias}

1. A merican Thoracic Society. Standardization of spirometry, 1994 update. Am J Respir Crit Care Med 1995;152:1107-1136.

2. American Thoracic Society. Lung function testing: Selection of reference values and interpretative strategies. Am Rev Respir D is 1991;144: 1202-1218.

3. Becklake MR. Concepts of normality applied to the measurement of lung function. Am J Med 1986;80:1158-1164.

4. Enright PL, Johnson LR, Connett JE, Voelker H, Buist S. Spirometry in the lung health study. Methods and quality control. Am Rev Respir Dis 1991; 143:1215-1223.

5. Enright PL. Surveillance for lung disease. Q uality assurance using computers and a team approach. 0 ccup Med 1992;7:209-225.

6. Vollmer W M, Johnson LR, MCC amant LE, Buist S. Methodologic issues in the analysis of lung function data. J Chron D is 1987;40:1013-1023.

7. Knudson RJ, Lebowitz MD, Holberg CJ, Burrows B. Changes in the normal maximal expiratory flow-volume curve with growth and aging. Am Rev Respir Dis 1983;127:725-734.

8. Q uanjer PH, ed. Standardized lung function testing. Bull Eur Physiopathol Resp 1983;19:suplemento 5:1-95.

9. Hankinson JL, O dencrantz JR, Fedan KB. Spirometric reference values from a sample of the general U.S. population. Am J Respir C rit C are Med 1999;159:179-187.

10. 0 yarzun M. N ormal spirometric values in the Chilean population. Rev Med Chil 1996;124:1365-1367.

11. Pérez-Padilla JR e investigadores del proyecto EMPECE. Metodología de la realización de espirometrías en el proyecto EMPECE. LXXXIV Reunión Reglamentaria de la A sociación de Investigación Pediátrica; 1997 6-7 de junio; Santa Cruz, Tlaxcala, México. 